OPEN ACCESS

Edited by:

Philipp Albrecht,

Heinrich Heine University of

Düsseldorf, Germany

Reviewed by:

Ingo Kleiter,

Marianne-Strauss-Klinik, Germany

Ilya Ayzenberg,

Ruhr University Bochum, Germany

${ }^{*}$ Correspondence:

Xiang Gao

xiang.gao7915@gmail.com

Specialty section:

This article was submitted to

Multiple Sclerosis and

Neuroimmunology,

a section of the journal

Frontiers in Neurology

Received: 16 April 2021

Accepted: 30 August 2021 Published: 03 November 2021

Citation:

Singh P, Gao X, Kleijn HJ, Bellanti F and Pelto $R$ (2021) Eculizumab

Pharmacokinetics and

Pharmacodynamics in Patients With Neuromyelitis Optica Spectrum Disorder. Front. Neurol. 12:696387.

doi: 10.3389/fneur.2021.696387

\section{Eculizumab Pharmacokinetics and Pharmacodynamics in Patients With Neuromyelitis Optica Spectrum Disorder}

\author{
Pratap Singh ${ }^{1}$, Xiang Gao ${ }^{2 *}$, Huub Jan Kleijn ${ }^{3}$, Francesco Bellanti ${ }^{3}$ and Ryan Pelto ${ }^{2}$ \\ ${ }^{1}$ Department of Clinical Pharmacology, Alexion Pharmaceuticals Inc., Boston, MA, United States, ${ }^{2}$ Department of \\ Pharmacometrics, PKJPD M\&S, Clinical Development and Translational Sciences, Alexion Pharmaceuticals Inc., Boston, MA, \\ United States, ${ }^{3}$ Certara Strategic Consulting, Oss, Netherlands
}

Objective: To investigate the pharmacokinetics and pharmacodynamics of the approved 900/1,200 mg dosing regimen for the terminal complement component 5 (C5) inhibitor eculizumab in patients with neuromyelitis optica spectrum disorder (NMOSD).

Methods: Data were analyzed from 95 patients with aquaporin-4-lgG-positive NMOSD who received eculizumab during the PREVENT study (ClinicalTrials.gov: NCT01892345). Relationships were explored between eculizumab exposure and free complement C5 concentrations, terminal complement activity, and clinical outcomes.

Results: Pharmacokinetic data were well-described by a two-compartment model with first-order elimination, and time-variant body-weight and plasmapheresis/plasma exchange effects. Steady-state serum eculizumab concentrations were achieved by Week 4 and were sustained, with serum trough eculizumab concentrations maintained above the $116 \mu \mathrm{g} / \mathrm{ml}$ threshold for complete complement inhibition throughout 168 weeks of treatment in all post-baseline samples from 89\% of patients. Complete inhibition of terminal complement was achieved at Day 1 peak and pre-dosing trough eculizumab concentration in nearly all post-baseline samples assessed (free C5 $<0.5 \mu \mathrm{g} / \mathrm{ml}$ in all post-baseline samples from 96\% of patients; in vitro hemolysis $<20 \%$ in all post-baseline samples from $93 \%$ of patients). Kaplan-Meier survival analysis of time to first relapse showed separation of eculizumab-treated patients from those receiving placebo, but no separation based on eculizumab exposure quartile, indicating an optimized dose regimen with maximized efficacy.

Conclusions: The approved eculizumab dosing regimen $(900 / 1,200 \mathrm{mg})$ for adults with aquaporin-4-lgG-positive NMOSD is confirmed by rigorous quantitative model-based analysis of exposure-response. The data demonstrate that eculizumab's mechanism of action translates into clinical effect by achieving rapid, complete, and sustained terminal complement inhibition.

Keywords: neuromyelitis optica spectrum disorder, pharmacokinetics, pharmacodynamics, complement, eculizumab, autoimmune, exposure-response analysis 


\section{INTRODUCTION}

Neuromyelitis optica spectrum disorder (NMOSD) is a rare autoimmune disorder of the central nervous system (CNS), predominantly affecting the optic nerves and spinal cord, and characterized by severe and unpredictable attacks (relapses). These relapses can cause disabling neurologic damage, including weakness, numbness, bowel/bladder dysfunction, pain, paralysis, and blindness $(1,2)$. The damage is irreversible and its accumulation increases overall mortality (3-6).

The majority of patients with NMOSD (62-88\%) test positive for autoantibody immunoglobulin (Ig)G to the water-channel protein aquaporin-4 (AQP4), which is expressed predominantly on the endfeet processes of astrocytes in the CNS (3, 7-9). The binding of anti-AQP4-IgG is one of the key underlying causes of neurologic damage in NMOSD, as the autoantibodies are predominantly of IgG1 subclass and induce activation of the complement cascade. It is thought that anti-AQP4-IgG is generated in the periphery and crosses the blood-brain barrier by passive diffusion from serum $(9,10)$. The first indication of the role of complement in the pathophysiology of NMOSD came with the demonstration of deposits of perivascular complement components and evidence for the formation of the membrane attack complex in demyelinating lesions in patients with NMOSD (11). Subsequent in vitro, animal-model, and clinical studies have provided evidence for activation of the complement pathway in response to the binding of anti-AQP4IgG to astrocytic AQP4 in the CNS (12-15). The activation results in cleavage of complement protein $\mathrm{C} 5$ into $\mathrm{C} 5 \mathrm{a}$ (which promotes inflammation) and $\mathrm{C} 5 \mathrm{~b}$ (which coordinates formation of the membrane attack complex); increased concentrations of terminal complement components (C5a and sC5b-9) have been demonstrated in the cerebrospinal fluid (CSF) of patients with active $\operatorname{NMOSD}(12,15)$. The complement activity rapidly causes damage to astrocytes, as well as permeabilization of the blood-brain barrier, ultimately leading to astrocyte necrosis, demyelination, and neuronal death (11, 16-19).

As complement activation is one of the major determinants of disease pathogenesis in patients with NMOSD, inhibition of terminal complement activation is an effective therapeutic approach $(14,17,20,21)$. This was confirmed in the Phase 3, randomized, double-blind, placebo-controlled, time-to-event PREVENT study in 143 adults with AQP4-IgG-positive NMOSD, in which treatment with eculizumab-a terminal complement inhibitor-significantly reduced the risk of relapse by $94 \%$ vs. placebo $(p<0.001)$. Based on results from this study, eculizumab was approved in 2019 in the US, Europe, Japan, and Canada, and in 2020 in Australia, for the treatment of NMOSD in adult patients who are anti-AQP4 antibody positive (22-27).

Eculizumab is a novel, humanized IgG2/4 monoclonal antibody that inhibits terminal complement activation by selectively binding with high affinity to complement component $\mathrm{C} 5$, inhibiting its cleavage into $\mathrm{C} 5 \mathrm{a}$ and $\mathrm{C} 5 \mathrm{~b}$ and the subsequent neurologic damage $(8,28)$. It does not interact with earlier (proximal) components in the complement-activation pathway thus preserving complement functioning at that level, which is required for opsonization and immune complex clearance
$(29,30)$. Eculizumab was derived from a murine antihuman C5 monoclonal antibody and engineered to reduce immunogenicity and the potential to elicit proinflammatory and antigenic responses by substituting with human IgG2 and IgG4 components, which, compared with IgG1 and IgG3, have low potential for activating the complement cascade (29).

Using data from the PREVENT study, the aim of the current analysis was to further characterize the pharmacokinetic and pharmacodynamic properties of eculizumab in patients with AQP4-IgG-positive NMOSD to confirm the rationale, efficacy, and safety of the approved eculizumab dosing regimen in this indication. A model-based approach was used to characterize the population-pharmacokinetic parameters of eculizumab and between-subject variability, to assess potential factors impacting eculizumab pharmacokinetics, and to explore pharmacokinetic/pharmacodynamic relationships for free serum and CSF concentrations and terminal complement activity. Exposure-response relationships were also assessed, as well as the potential immunogenicity of eculizumab.

\section{METHODS}

\section{Study Population and Study Design}

This analysis was based on pharmacokinetic, pharmacodynamic, efficacy, and safety data from the randomized, double-blind, placebo-controlled, multicenter, Phase 3 PREVENT study (ClinicalTrials.gov: NCT01892345) of eculizumab in patients with AQP4-IgG-positive NMOSD. The primary endpoint was time to first adjudicated on-trial relapse. Clinical efficacy and safety results have been reported (31).

The PREVENT study population comprised 143 adult patients with AQP4-IgG-positive NMOSD, of whom 96 patients received the now approved dosing regimen of intravenous eculizumab $900 \mathrm{mg}$ weekly (every $7 \pm 2$ days) for the first four doses (starting on Day 1), followed by 1,200 mg 1 week later at Week 4 (the fifth dose) and then every 2 weeks ( $14 \pm 2$ days) thereafter.

The study was conducted in accordance with the Declaration of Helsinki (32), the International Conference on Harmonisation Guidelines for Good Clinical Practice (33), and applicable regulatory requirements. It was approved by the institutional review board at each participating institution. All the patients provided written informed consent before participation.

\section{Sample Collection}

Each intravenous eculizumab infusion was administered over $\sim 35 \mathrm{~min}$. Blood samples for measurement of baseline and trough eculizumab concentrations, anti-AQP4 antibody titer, serum free C5 concentrations, and hemolytic activity were taken 5-90 $\mathrm{min}$ before infusion of eculizumab (trough concentration; $\mathrm{C}_{\text {trough }}$ ) at baseline, Weeks 4,8 , and 12, and then every 12th week until the end of the study or early termination. Blood samples for analysis of peak eculizumab concentration $\left(\mathrm{C}_{\max }\right)$, free C5 concentrations, and hemolytic activity were taken at least $60 \mathrm{~min}$ after the completion of eculizumab infusion at Day 1, Weeks 4, 8, and 12 , and then every 12 th week.

CSF samples (baseline and trough only) for measurement of eculizumab and free $\mathrm{C} 5$ concentrations were available from those 
patients who consented to lumbar puncture procedures and were taken at baseline, Weeks 12 and 24, and then every 24th week until the end of the study or early termination.

Blood samples for measurement of anti-drug antibodies (ADAs) and neutralizing antibodies (NAbs) were taken 590 min before infusion of eculizumab at baseline, Weeks 4 and 12 , and then every 12 th week until the end of the study or early termination.

\section{Sample Analyses}

Analyses of eculizumab and free C5 in serum and CSF, and of in vitro hemolytic activity were conducted by Charles River Laboratories (Mattawan, MI, USA) using laboratory-developed tests, validated according to the contemporary recommendations of the US Food and Drug Administration (34) and the European Medicines Agency (35).

\section{Pharmacokinetics of Eculizumab in Serum and CSF}

Total (bound plus free) concentrations of eculizumab in serum and CSF (where samples were available) were measured by enzyme-linked immunosorbent assays with quantification ranges of $9.38-600 \mu \mathrm{g} / \mathrm{ml}$ and $0.00309-0.400 \mu \mathrm{g} / \mathrm{ml}$, respectively. Serum analysis used absorbance detection, while CSF analysis used electrochemiluminescence [Meso Scale Discovery (MSD) platform; Meso Scale Diagnostics, Rockville, MD, USA].

\section{Anti-AQP4 Antibody Titer}

Anti-AQP4 autoantibody titers were measured using a fluorescence-activated cell sorting assay (Mayo Clinic Laboratories, Rochester, MN, USA).

\section{Free C5 Concentration}

Concentrations of free C5 in serum (as a measure of target engagement) and in CSF (to determine CNS penetration) were measured by ligand-binding assays using eculizumab for free C5 capture and chemiluminescent detection (MSD platform). Quantification ranges in serum and CSF were $0.0274-20 \mu \mathrm{g} / \mathrm{ml}$ and $3.00-400 \mathrm{ng} / \mathrm{ml}$, respectively. Serum free C5 $<0.5 \mu \mathrm{g} / \mathrm{ml}$ represents complete inhibition of terminal complement (Alexion Pharmaceuticals, data on file).

\section{In vitro Hemolytic Activity}

Complement-mediated hemolytic activity of patient serum was measured in vitro by lysis of chicken red blood cells (cRBCs) using a semi-quantitative assay. cRBCs were sensitized with an anti-cRBC polyclonal antibody, incubated with serum, centrifuged and the supernatant measured using absorbance $(415 \mathrm{~nm})$ for hemoglobin released via cell lysis. Percent hemolysis was expressed relative to pooled normal human serum, as proof of the pharmacology of eculizumab. cRBC hemolysis $<20 \%$ represents complete terminal complement inhibition (Alexion Pharmaceuticals, data on file).

\section{Immunogenicity}

To investigate the presence or absence of an immune response to eculizumab, serum concentrations of ADAs against eculizumab were measured using a solution-phase bridging assay with electrochemiluminescent detection (MSD platform).
The method used a tiered approach, with screening (sensitivity $6.26 \mathrm{ng} / \mathrm{ml}$ ) and confirmatory competition steps to identify samples as positive or negative for the presence of ADAs to eculizumab. If ADAs to eculizumab were detected, the positive ADA samples were analyzed further for titer, and for the presence of NAbs via a ligand-binding assay for inhibition of $\mathrm{C} 5$ binding with electrochemiluminescent detection (MSD platform).

\section{Population-Pharmacokinetic Methods}

A previously developed model of eculizumab population pharmacokinetics in patients with generalized myasthenia gravis (gMG) (36) was used as the base model for full evaluation of the impact of covariates on eculizumab pharmacokinetics in patients with AQP4-IgG-positive NMOSD. As described in the accompanying paper (36), a two-compartment model with firstorder elimination was developed to describe the pharmacokinetic profile of eculizumab in patients with gMG, using serum eculizumab concentration data from the REGAIN study (37). In that model, random effects were included for central clearance (CL) and central volume of distribution $\left(\mathrm{V}_{1}\right)$. A proportional error was used to describe the residual variability of the study data. All pharmacokinetic parameters were allometrically scaled by baseline body weight. Eculizumab CL was estimated with body weight as a covariate at $\sim 0.00749 \mathrm{~L} / \mathrm{h}$ for a typical patient with gMG weighing $70 \mathrm{~kg}$. Plasmapheresis/plasma exchange (PLEX) events were modeled to account for a temporary increase in eculizumab CL during the PLEX administration period.

Model development for population pharmacokinetics in patients with AQP4-IgG-positive NMOSD was carried out in a stepwise manner using first-order, conditional estimation with interaction. Body weight, centered on $70 \mathrm{~kg}$, was considered a structural factor on CL and intercompartmental clearance (Q) and on $\mathrm{V}_{1}$ and peripheral volume of distribution $\left(\mathrm{V}_{2}\right)$ parameters. PLEX was considered a structural factor on CL. Body weight and PLEX factors were included early in model development before full covariate evaluation; therefore, they were not part of the covariate searching procedure. The structural pharmacokinetic model (including body weight) was defined as the base model and served as the starting point for covariate assessment.

Post-hoc pharmacokinetic parameter random effects (ETAs) obtained from the base structural model were used in an exploratory analysis to identify potential covariatepharmacokinetic parameter relationships for the formal covariate search.

In order to assess whether dose alterations would be required in particular patient populations, a number of baseline covariates were evaluated, including demographic and clinical factors (see Supplementary Methods for full list). Covariates were identified using a stepwise covariate modeling procedure (see Supplementary Methods). The criteria that guided development of the model that best described eculizumab disposition are detailed in the Supplementary Methods. Model performance and robustness were evaluated by visual predictive check and non-parametric bootstrap analyses. 


\section{Exploratory Analysis of Free C5 and Hemolytic Activity}

Exploratory plots were generated and summary statistics calculated for free $\mathrm{C} 5$ and hemolytic activity by clinic visit. Because complete inhibition was achieved in almost all patients who received eculizumab, the data were insufficient for development of pharmacokinetic/pharmacodynamic models for either free C5 or hemolytic activity. The previously established threshold eculizumab concentration to achieve complete terminal complement inhibition [see accompanying paper (36)] was used as a benchmark to assess the relationship between eculizumab exposures and pharmacodynamic endpoints.

\section{Exposure-Response for Efficacy}

The efficacy analysis included all eculizumab-treated patients for whom post-hoc pharmacokinetic parameters were available. Kaplan-Meier plots of relapse-free survival were generated for the primary endpoint-time to first adjudicated on-trial relapse. For eculizumab-treated patients, time to first adjudicated on-trial relapse was evaluated with respect to quartiles of eculizumab exposure [eculizumab area under the concentration-time curve within one dosing interval, i.e., 2 weeks, at steady state $\left.\left(\mathrm{AUC}_{\mathrm{ss}}\right)\right]$ values predicted from the population-pharmacokinetic model.

\section{Exposure-Response for Safety}

Treatment-emergent adverse events (AEs) of special interestdefined as infections (meningococcal infections, Aspergillus infections, other serious infections, or sepsis), infusion reactions, serious cutaneous adverse reactions, cardiac disorders, and angioedema-and treatment-emergent AEs that occurred in $\geq 5 \%$ of eculizumab-treated patients were evaluated by treatment and by eculizumab exposure quartile. Individual eculizumab $\mathrm{AUC}_{\mathrm{ss}}$ values were derived from post-hoc exposure estimates and were used as the exposure metric.

\section{Subgroup Analysis}

Subgroup analyses were undertaken in Asian and Japanese patients.

\section{RESULTS}

A total of 96 patients received eculizumab treatment during the PREVENT study; pharmacokinetic data were available for 95 patients, of whom 37 were Asian and nine were Japanese. The numbers of patients with data available for each analysis are shown in Table 1. Patient characteristics, clinical efficacy, and safety results from the PREVENT study have been reported previously (31). In brief, the mean [standard deviation (SD)] baseline characteristics of the 96 eculizumab-treated patients were: age 43.8 (13.4) years; body weight 68.4 (20.2) kg; body mass index $25.8(7.0) \mathrm{kg} / \mathrm{m}^{2}$; estimated glomerular filtration rate $114.2(37.9) \mathrm{ml} / \mathrm{min} / 1.73 \mathrm{~m}^{2}$; white blood cell count $6.5(2.2) \times$ $10^{9} / \mathrm{L}$; and albumin $43.1(3.1) \mathrm{g} / \mathrm{L}$. Of the 96 patients, $46(48 \%)$ were White, 37 (39\%) were Asian, nine (9\%) were Black/African American, and four (4\%) were Other; there were nine patients (9\%) of Japanese descent. The mean (SD) annualized relapse rate 24 months before screening was 1.9 (0.9) relapses/year. Fifteen
TABLE 1 | Numbers of patients with data available for the analyses.

\begin{tabular}{lc} 
Analysis & Number of patients \\
\hline Received eculizumab & 96 \\
Population-PK (eculizumab serum concentration) & $94^{\mathrm{a}, \mathrm{b}}$ \\
Serum free C5 & $95^{\mathrm{a}}$ \\
Percent CRBC hemolysis & $95^{\mathrm{a}}$ \\
CSF eculizumab concentration & $4^{\mathrm{C}}$ \\
CSF free C5 & $4^{\mathrm{c}}$ \\
Population-PK: efficacy & $95^{\mathrm{a}, \mathrm{b}}$ \\
Population-PK: safety & $95^{\mathrm{a}, \mathrm{b}}$ \\
& \\
\hline C5, complement component 5; cRBC, chicken red blood cell; CSF, cerebrospinal fluid; \\
PK, pharmacokinetic. \\
aPopulation-PK, serum free C5, and hemolytic-activity data were not available for one \\
patient because of a local protocol requirement; this patient was excluded from the \\
population-PK analysis but was included in the efficacy and safety exposure-response \\
analyses as post-hoc exposure was predicted by the PK model. \\
bPopulation-PK data were not available for one patient because of an unusual PK profile; \\
no post-hoc PK parameters were obtained for this patient and, therefore, data for this \\
patient were also excluded from the efficacy and safety exposure-response analyses. \\
cEight eculizumab-treated patients provided informed consent for CSF sampling; \\
however, CSF samples at scheduled visits after the first dose were only available for four \\
eculizumab-treated patients.
\end{tabular}

PLEX events were recorded for three patients (four, five, and six events, respectively) treated with eculizumab during the study.

\section{Pharmacokinetics}

\section{Serum Eculizumab Concentrations}

Following implementation of the eculizumab 900/1,200 mg dosing regimen, steady-state serum eculizumab concentrations were achieved by Week 4 and were sustained throughout the treatment period. Eculizumab concentrations were above the previously established threshold of $116 \mu \mathrm{g} / \mathrm{ml}$ for complete complement inhibition (36) in $96.7 \%$ of all post-baseline samples. At any timepoint studied, $92-100 \%$ of patients in whom concentrations were measured had $\mathrm{C}_{\text {trough }}$ values above the threshold. Serum eculizumab $\mathrm{C}_{\text {trough }}$ values were maintained well above the $116 \mu \mathrm{g} / \mathrm{ml}$ threshold in all post-baseline samples from the majority of patients $(85 / 95 ; 89 \%)$ (Figure 1). None of the 10 patients who at any time recorded below-threshold $\mathrm{C}_{\text {trough }}$ concentrations had received PLEX; however, in six of these patients, the blood sample(s) was taken following a delay in the scheduled eculizumab administration (i.e., eculizumab administration, and the pre-dose blood sampling, occurred more than 14 days after the last dose).

Between Weeks 4 and 168, the mean (SD) $\mathrm{C}_{\max }$ by visit ranged from 850 (297) to 930 (338) $\mu \mathrm{g} / \mathrm{ml}$. Median serum eculizumab $\mathrm{C}_{\text {trough }}$ was $\sim 400 \mu \mathrm{g} / \mathrm{ml}$, with the majority of individual $\mathrm{C}_{\text {trough }}$ values ranging from 100 to $700 \mu \mathrm{g} / \mathrm{ml}$. Between Weeks 4 and 168 , the mean (SD) $\mathrm{C}_{\text {trough }}$ by visit ranged from 378 (167) to 432 (169) $\mu \mathrm{g} / \mathrm{ml}$.

A simulation of the steady-state eculizumab serum concentrations found that eculizumab administration up to 2 days after the scheduled 2-week timepoint maintained $\mathrm{C}_{\text {trough }}$ values above the $116 \mu \mathrm{g} / \mathrm{ml}$ threshold in almost all patients (data not shown). 


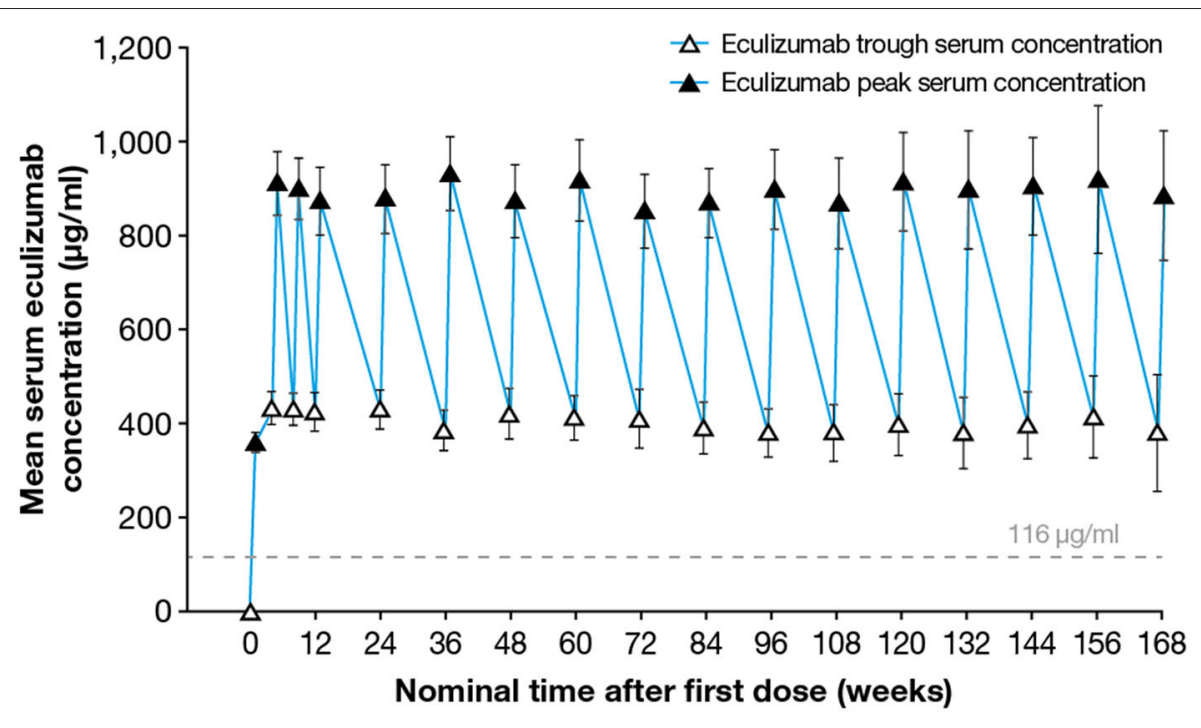

$\begin{array}{lllllllllllllll}\text { Peak (n) } & 86-94 & 81 & 74 & 64 & 58 & 57 & 50 & 43 & 32 & 28 & 24 & 19 & 17 & 12 \\ \text { Trough (n) } & 88-93 & 81 & 74 & 65 & 58 & 57 & 51 & 43 & 33 & 30 & 24 & 20 & 17 & 12\end{array}$

FIGURE 1 | Serum eculizumab concentrations during the study. Mean (95\% confidence interval) trough and peak serum eculizumab concentrations in patients who received eculizumab in the PREVENT study; eculizumab concentrations below the lower limit of quantification $(9.38 \mu \mathrm{g} / \mathrm{ml}) \mathrm{were}$ analyzed as $4.69 \mu \mathrm{g} / \mathrm{ml}$ (half the lower limit of quantification). Eculizumab concentrations above $116 \mu \mathrm{g} / \mathrm{ml}$ (dashed line) indicate sufficient concentration to achieve complete complement inhibition.

\section{CSF Eculizumab Concentrations}

Post-first dose CSF samples taken at scheduled visits were available for four eculizumab-treated patients. The mean timematched serum eculizumab concentrations were 402- to 649-fold higher than CSF eculizumab concentrations. In three patients, CSF eculizumab $C_{\text {trough }}$ values were close to $1 \mu \mathrm{g} / \mathrm{ml}$, while the fourth patient showed a CSF $\mathrm{C}_{\text {trough }}$ value 3- to 4-fold higher than those of the other patients. Within each patient, the CSF $\mathrm{C}_{\text {trough }}$ values remained stable over time.

\section{Population-Pharmacokinetic Model}

The two-compartment model with first-order elimination developed for the analysis of eculizumab pharmacokinetics in patients with gMG was used as the base model. However, a number of modifications were implemented. To account for the extended treatment duration in the NMOSD study (data were analyzed for the first 168 weeks of treatment, whereas treatment duration was 26 weeks in the MG study), body weight was included as a time-varying covariate, rather than as baseline body weight. Additionally, an additive effect of PLEX on CL, rather than a multiplicative effect as used in the original model, was found to improve the individual fit of the model for patients undergoing PLEX procedures.

The initial exploration of potential covariate effects showed no trends between any of the covariates of interest and CL or $V_{1}$, except for effects of the baseline serum albumin concentration and the patient's sex on $V_{1}$, which were the only two covariates showing a $p$-value close to 0.01 . However, following stepwise forward addition and backward elimination steps to determine the contribution of these covariates, neither met the criteria to be retained in the model.
In the final population-pharmacokinetic model, the eculizumab pharmacokinetic data from patients with NMOSD were well-described by a two-compartment model with firstorder elimination. The final model parameter estimates showed good precision with percent standard error of estimation always below $26 \%$ and acceptable inter-individual variability (ETA) shrinkage. Parameter estimates for the final populationpharmacokinetic model are provided in Supplementary Table 1. Bayesian estimates of the pharmacokinetic parameters are summarized in Table 2: the mean (SD) terminal half-life was estimated to be $414(103) \mathrm{h}$, and the mean (SD) CL was estimated to be $0.00759(0.00313) \mathrm{L} / \mathrm{h}$.

Random effects were included for $\mathrm{CL}$ and $\mathrm{V}_{1}$ and a correlation term was estimated between the two parameters. All pharmacokinetic parameters were allometrically scaled by time-varying body weight; in addition, PLEX events were modeled to account for a temporary increase in eculizumab CL during the PLEX period. Eculizumab pharmacokinetic values were not influenced by any of the other covariates examined.

No differences were observed in steady-state eculizumab exposure parameters AUC, $\mathrm{C}_{\max }$, and $\mathrm{C}_{\text {trough }}$ between Asian and non-Asian patients or between Japanese and non-Japanese patients, after normalization for body weight (Figure 2). Further details of eculizumab pharmacokinetic parameters in Asian and non-Asian patients, and in Japanese and non-Japanese patients are provided in Supplementary Tables 2, 3, respectively.

\section{Immunogenicity}

Anti-eculizumab immunogenicity was negligible, with two ADA-positive samples, one each from two patients. 
TABLE 2 | Summary of estimated pharmacokinetic parameters.

\begin{tabular}{|c|c|c|c|c|c|c|c|}
\hline Statistic & Mean & SD & Median & 2.5th percentile & 97.5th percentile & Minimum & Maximum \\
\hline CL (L/h) & 0.00759 & 0.00313 & 0.00668 & 0.00418 & 0.0154 & 0.00362 & 0.0204 \\
\hline$V_{1}(L)$ & 2.33 & 0.527 & 2.21 & 1.58 & 3.4 & 1.56 & 3.68 \\
\hline$V_{2}(L)$ & 2.11 & 0.367 & 2.06 & 1.56 & 2.9 & 1.49 & 3.09 \\
\hline Q (L/h) & 0.238 & 0.0638 & 0.226 & 0.148 & 0.382 & 0.138 & 0.42 \\
\hline$C_{\max , s s}(\mu \mathrm{g} / \mathrm{ml})$ & 928 & 241 & 898 & 525 & 1,380 & 453 & 1,530 \\
\hline $\mathrm{C}_{\text {trough,ss }}(\mu \mathrm{g} / \mathrm{ml})$ & 405 & 152 & 416 & 140 & 706 & 92.7 & 811 \\
\hline Terminal half-life ${ }^{a}(h)$ & $414^{b}$ & 103 & 438 & 238 & 641 & 202 & 765 \\
\hline $\mathrm{AUC}_{\mathrm{ss}}(\mu \mathrm{g} \cdot \mathrm{h} / \mathrm{ml})$ & 180,000 & 57,900 & 180,000 & 77,900 & 287,000 & 58,700 & 331,000 \\
\hline Accumulation ratio & 2.06 & 0.381 & 2.05 & 1.32 & 2.8 & 1.19 & 3.28 \\
\hline
\end{tabular}

Data are summaries of the individual Bayesian estimates.

a Terminal half-life was calculated as: $\left(V_{1}+V_{2}\right) / C L * \ln (2)$.

b Terminal half-life mean was calculated as harmonic mean.

AUC, area under the concentration-time curve within one dosing interval; $C L$, clearance; $C_{\max }$, peak concentration; $C_{\text {trough }}$, concentration at the end of the dosage interval; $Q$, intercompartmental clearance; SD, standard deviation; ss, steady state; $V_{1}$, volume of distribution in the central compartment; $V_{2}$, volume of distribution in the peripheral compartment.

ADA titers for these two patients were low and transient; their subsequent samples were negative on ADA screening. There was no impact on observed pharmacokinetic/pharmacodynamic data in either patient, and neither patient experienced relapse of NMOSD. There were no NAb-positive samples.

\section{Pharmacodynamics}

The eculizumab threshold concentration to achieve complete terminal complement inhibition $(116 \mu \mathrm{g} / \mathrm{ml})$ has been established using populationpharmacokinetic/pharmacodynamic data from the REGAIN study in patients with gMG (36). Given the consistency of eculizumab pharmacokinetics/pharmacodynamics across indications, this threshold was used as a benchmark to assess the relationship between eculizumab exposure and the pharmacodynamic endpoint.

\section{Serum Free C5 Concentrations}

At any post-baseline timepoint studied, $97-100 \%$ of patients in whom concentrations were measured had free $\mathrm{C} 5$ concentrations $<0.5 \mu \mathrm{g} / \mathrm{ml}$. Serum free C5 concentrations $<0.5 \mu \mathrm{g} / \mathrm{ml}$ were observed in $99.3 \%$ of post-baseline samples from eculizumabtreated subjects, and in all post-baseline samples (observed at Day 1 eculizumab $\mathrm{C}_{\max }$ and at all visits at the time of eculizumab $C_{\text {trough }}$ ) from 91/95 (96\%) of eculizumab-treated patients (Figure 3A). In the remaining patients, serum free $\mathrm{C} 5$ concentrations $<0.5 \mu \mathrm{g} / \mathrm{ml}$ were seen at the time of $\mathrm{C}_{\text {trough }}$ at the majority of visits during the maintenance phase of treatment, except for one patient who consistently showed serum free C5 concentrations $>0.5 \mu \mathrm{g} / \mathrm{ml}$ for all samples available up to Week 36. The relationship between eculizumab exposure and free C5 concentration confirms that at the exposure range achieved in the study, serum free C5 concentrations were reduced below the $0.5 \mu \mathrm{g} / \mathrm{ml}$ threshold for complete inhibition of terminal complement (Figure 4A).

\section{CSF Free C5 Concentrations}

Pre-first-dose CSF samples were available for eight eculizumabtreated patients (median free C5 concentration $253 \mathrm{ng} / \mathrm{ml}$, SD $108 \mathrm{ng} / \mathrm{ml}$ ). Post-first-dose CSF samples at scheduled visits were available for four eculizumab-treated patients. All post-firstdose CSF free C5 concentrations were below the lower limit of quantification of $3.00 \mathrm{ng} / \mathrm{ml}$, indicating complete terminal complement inhibition in CSF.

\section{Hemolysis}

At any timepoint studied, $90-100 \%$ of patients in whom concentrations were measured demonstrated complete inhibition of terminal complement, as defined by $<20 \%$ in vitro $\mathrm{cRBC}$ hemolysis. Overall, $97.7 \%$ of all post-baseline samples achieved in vitro $\mathrm{cRBC}$ hemolysis $<20 \%$. Additionally, in vitro cRBC hemolysis $<20 \%$ was achieved at all time points (Day 1 eculizumab $\mathrm{C}_{\max }$ and at all visits at the time of eculizumab $\left.\mathrm{C}_{\text {trough }}\right)$ in nearly all treated patients $(88 / 95$; 93\%) (Figure 3B). The relationship between eculizumab exposure and hemolytic activity confirms that at the achieved eculizumab exposure range, hemolytic activity was reduced below the threshold of $20 \%$ for the large majority of assessments (Figure 4B). In a few samples ( $\sim 1.9 \%$ of the total) hemolytic activity was determined to be $\geq 20 \%$ when serum eculizumab concentration was $\geq 116 \mu \mathrm{g} / \mathrm{ml}$; this observation is likely to reflect assay variability.

\section{Exploratory Exposure-Response Analysis Time to First Adjudicated On-Trial Relapse}

Efficacy results have been reported previously (31). Adjudicated on-trial relapses occurred in 3/96 of eculizumab-treated patients (3\%) (Table 3). Complete complement suppression was achieved, based on serum free C5 and hemolysis data, in each of these three patients. Kaplan-Meier survival plots of time to first adjudicated on-trial relapse showed the treatment effect of the eculizumab 900/1,200 mg dose regimen. Consistent with the significant effect of eculizumab dosing on relapses, there was a clear separation in relapse-free survival across all exposure quartiles for eculizumab patients compared with patients in 


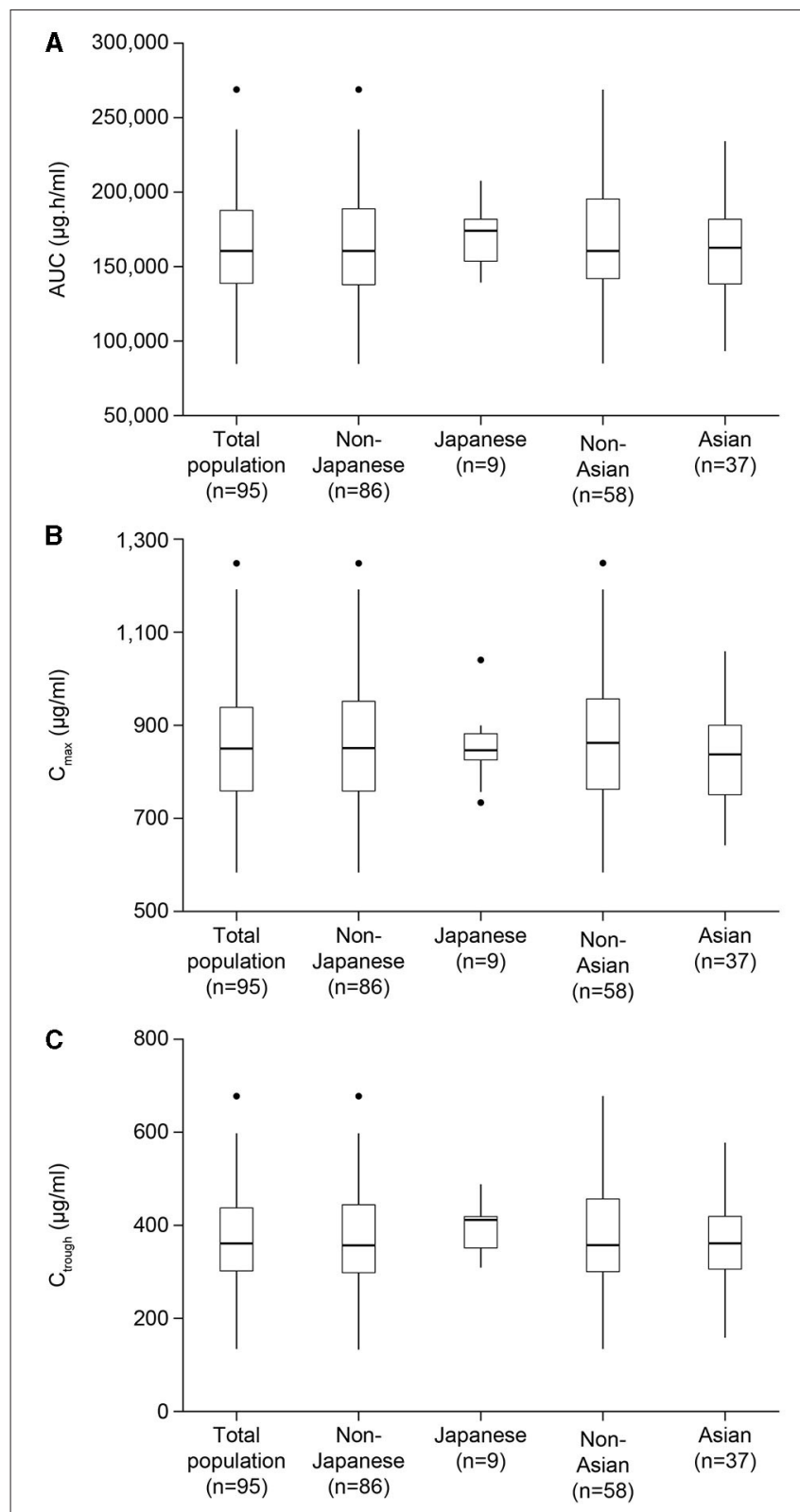

FIGURE 2 | Estimated body weight-normalized eculizumab exposure parameters for the total patient population, Asian, non-Asian, Japanese, and non-Japanese patients. Results are shown for parameters at steady state: (A) AUC, (B) $C_{\max }$, and (C) $C_{\text {trough. }}$. Exposures were normalized by body weight using the exponent estimated in the final model. AUC, area under the concentration-time curve within one dosing interval; $\mathrm{C}_{\max }$, peak concentration; $\mathrm{C}_{\text {trough }}$, concentration at the end of the dosage interval; ss, steady state.

the placebo group. Furthermore, it can be seen that there was no clear separation between the relapse-free survival curves across the different exposure quartiles, suggesting a maximal treatment effect was achieved within the observed exposure range (Figure 5). These findings were supported by data for on-trial relapses as determined by the treating physician (see Supplementary Material).

\section{Impact of Eculizumab Exposure on Anti-AQP4 Antibodies}

Eculizumab serum concentrations were similar across quartiles of anti-AQP4 antibody titers, consistent with the expectation that eculizumab concentrations would not impact anti-AQP4 autoantibody titers.

\section{Safety}

No trends were observed in AEs or AEs of special interest with increasing eculizumab exposure, comparing patients treated with eculizumab stratified in terms of the steady-state AUC quartiles (see Supplementary Table 5). Similarly, no meaningful safety trends were observed when comparing Asian vs. non-Asian patients and Japanese vs. non-Japanese patients. No differences were observed in AEs or AEs of special interest between eculizumab-treated patients and those who received placebo.

\section{DISCUSSION}

The results of this pharmacokinetic/pharmacodynamic analysis in patients with AQP4-IgG-positive NMOSD endorse the recommended dosing regimen for eculizumab (900 mg weekly for the first four doses, followed by $1,200 \mathrm{mg} 1$ week later and every 2 weeks thereafter). This analysis also verifies the association between eculizumab's mechanism of action and the efficacy demonstrated in clinical studies $(31,38)$.

Pharmacokinetic data were well-described by a twocompartment model with first-order elimination, based on a model developed previously to describe the pharmacokinetic profile of eculizumab in patients with gMG (36). It is of note that the pharmacokinetic model parameters are similar for patients with NMOSD and those with gMG; the typical CL and terminal half-life values of eculizumab for a person weighing $70 \mathrm{~kg}$ are almost identical in the two clinical conditions.

Sufficient serum concentrations of eculizumab were achieved from Day 1 of treatment, with complete terminal complement inhibition observed by the end of infusion of the first dose, and sustained throughout the entire treatment period in nearly all patients (serum free C5 $<0.5 \mu \mathrm{g} / \mathrm{ml}$ in $96 \%$ and in vitro $\mathrm{cRBC}$ hemolytic activity $<20 \%$ in $93 \%$ ). The rapid and sustained C5 suppression following eculizumab administration accounts for the significant treatment effect reported for the primary endpoint (first adjudicated on-trial relapse) and reduction in relapse risk observed in PREVENT (31). Complete complement suppression was achieved (based on serum free C5 concentration) in the three patients with adjudicated on-trial relapses who were treated with eculizumab, suggesting that other factor(s) may have triggered the relapse. It should be noted that complete terminal complement inhibition by eculizumab significantly reduced the risk of adjudicated relapse compared with placebo [hazard ratio 0.06 (95\% CI: 0.02, 0.20); $p<0.001$ ] (31).

The $\sim 17$-day terminal elimination half-life of eculizumab in patients with NMOSD allows a convenient interval of 2 weeks between doses, providing a small amount of leeway in dosing $( \pm 2$ days) without risking incomplete complement inhibition (free C5 $>0.5 \mu \mathrm{g} / \mathrm{ml}$ ). Consistent with the prescribing information to administer the maintenance dose of eculizumab within 2 days 


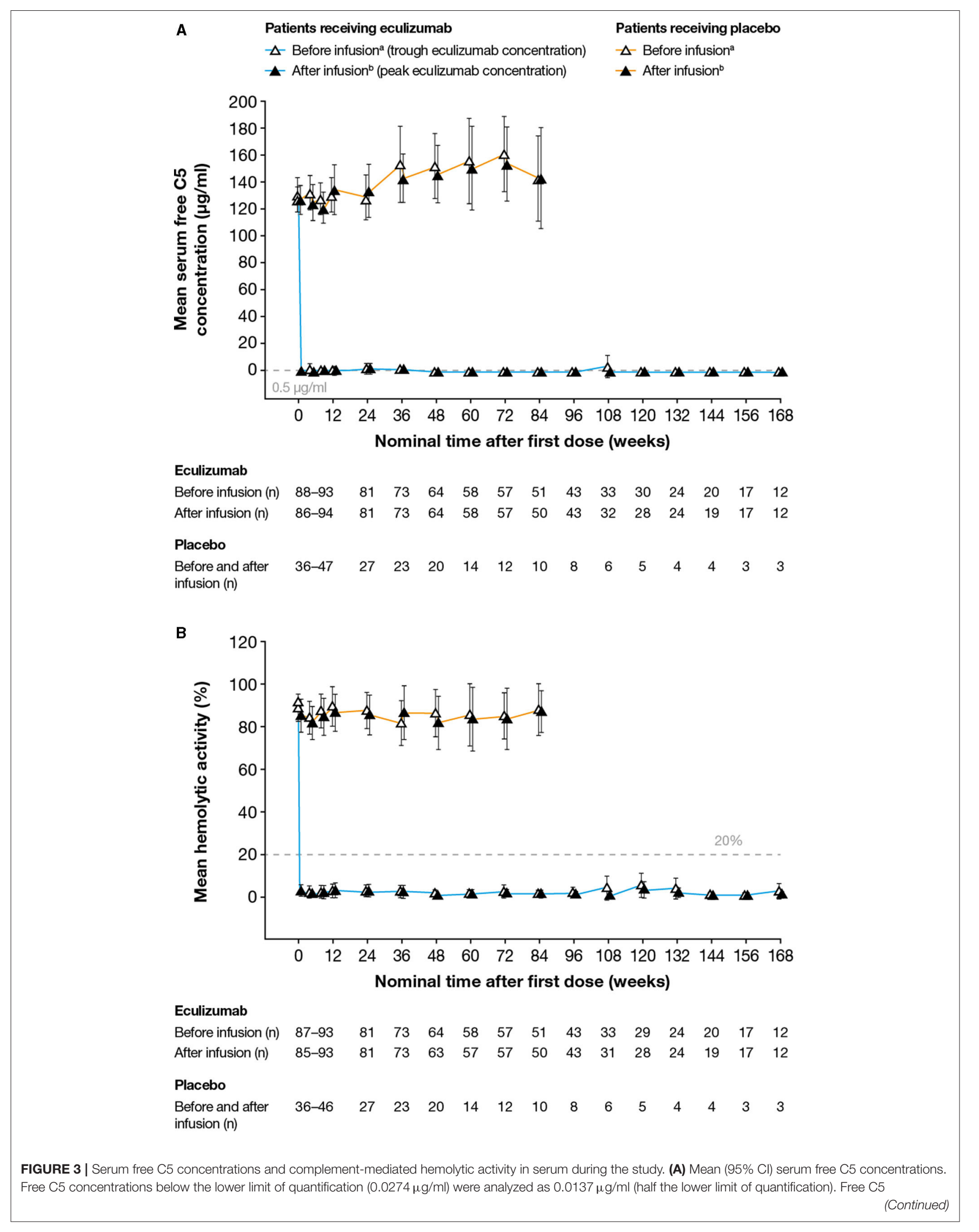


FIGURE 3 | concentrations below $0.5 \mu \mathrm{g} / \mathrm{ml}$ (dashed line) indicate complete terminal complement inhibition. No free C5 data were available for one patient. (B) Mean $(95 \% \mathrm{Cl})$ percent in vitro complement-mediated hemolytic activity of serum samples. Hemolysis values above $20 \%$ (dashed line) indicate incomplete inhibition of hemolysis. No hemolysis data were available for one patient. For both analyses, samples were taken before and after eculizumab infusion (i.e., at eculizumab serum trough and peak concentrations, respectively); samples taken before and after infusion in patients receiving placebo are shown for comparison. C5, complement protein 5; $\mathrm{Cl}$, confidence interval. a Samples taken 5-90 min before infusion. ${ }^{b}$ Samples taken 60 min after the completion of infusion. Data are plotted for timepoints when samples were available for $\geq 10$ patients. The numbers reported below the graphs are the numbers of patients for whom samples were tested at that timepoint.
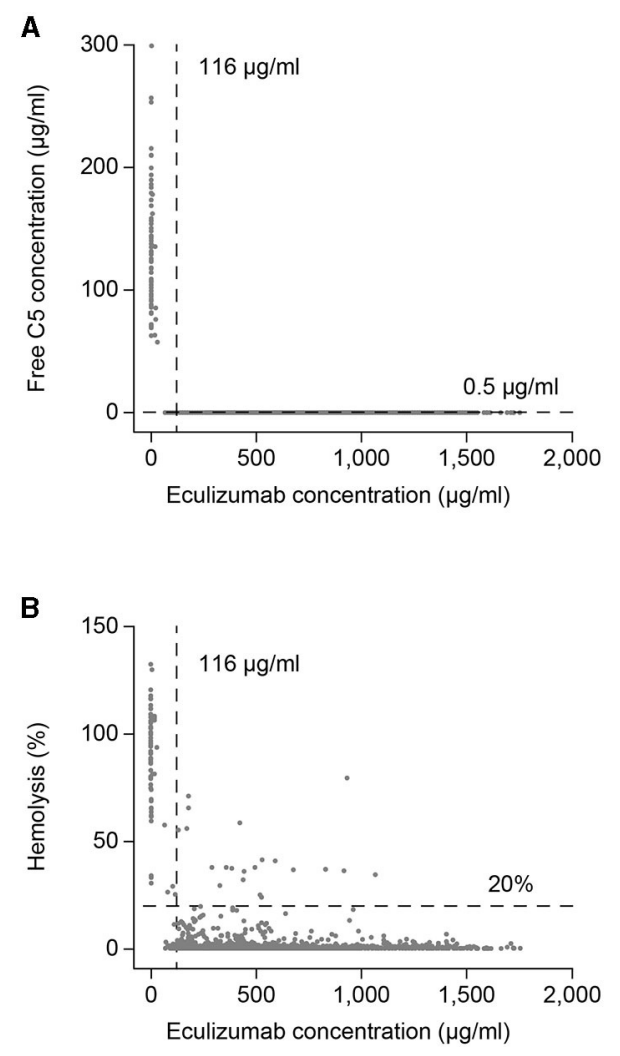

FIGURE 4 | Eculizumab exposure-response profiles for serum free C5 concentration and hemolytic activity. Scatterplots of (A) time-matched serum free C5 concentrations and eculizumab concentrations, and (B) time-matched percent hemolysis and eculizumab concentrations. Vertical lines at eculizumab $116 \mu \mathrm{g} / \mathrm{ml}$ and horizontal lines at free C5 $0.5 \mu \mathrm{g} / \mathrm{ml}$ in panel A and $20 \%$ hemolysis in panel $\mathrm{B}$ show thresholds representing complete terminal complement inhibition for serum eculizumab concentration, and for free C5 concentration and hemolytic activity, respectively. C5, complement component 5 .

of the 2-weekly recommended dosing timepoint in patients with NMOSD, simulation of the steady-state eculizumab serum concentrations suggests that administration within this time

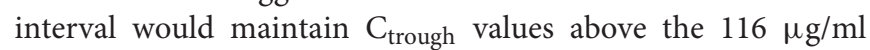
threshold in almost all patients. Day-to-day fluctuations in C5 concentrations are possible as a result of complementactivating events such as infection, surgery, and pregnancy; the eculizumab dosing regimen helps prevent C5 concentration increases translating into clinical relapse.

This analysis showed that in $89 \%$ of patients with AQP4-IgGpositive NMOSD, the 2 -week dosing interval provided sustained minimum serum eculizumab concentrations above the threshold $(116 \mu \mathrm{g} / \mathrm{ml})$ necessary to achieve complete terminal complement inhibition [as identified by exposure-response modeling in patients with gMG (36)]. In the remaining $12 \%$ of patients, the samples at the majority of timepoints showed eculizumab concentrations $>116 \mu \mathrm{g} / \mathrm{ml}$. Overall, $96.7 \%$ of the post-dose samples showed eculizumab concentrations $>116 \mu \mathrm{g} / \mathrm{ml}$. In addition, clinical efficacy was consistent across the range of steady-state serum eculizumab concentrations achieved in individual patients, confirming that the recommended dose has been optimized. This is consistent with the pharmacokinetic results from the Phase 3 study in patients with gMG (36).

The model confirmed that PLEX increased eculizumab clearance, which would require a temporary increase in dose during the plasma exchange period (39); fresh frozen plasma infusions are also likely to necessitate an eculizumab dose alteration due to the presence of complement proteins. Current recommendations $(22,23)$ are to administer a supplemental dose of eculizumab within $60 \mathrm{~min}$ after the end of each plasmapheresis/PLEX session, and $60 \mathrm{~min}$ before each infusion of fresh frozen plasma.

Although some pharmacokinetic parameters were affected by body weight, the data suggest that no dose adjustments

TABLE 3 | Number of patients with adjudicated on-trial relapse, by eculizumab exposure quartile.

\begin{tabular}{|c|c|c|}
\hline Group & $\begin{array}{c}\text { Total no. of } \\
\text { patients }\end{array}$ & $\begin{array}{l}\text { No. of patients with } \\
\text { adjudicated on-trial } \\
\text { relapse }\end{array}$ \\
\hline Patients receiving placebo & 47 & 20 \\
\hline \multicolumn{3}{|c|}{ Patients receiving eculizumab } \\
\hline $\begin{array}{l}\text { Patients with } \mathrm{AUC}_{\mathrm{Ss}} \text { within } \\
\text { 1st quartile (range } \\
58,714-143,644 \mu \mathrm{g} \cdot \mathrm{h} / \mathrm{ml} \text { ) }\end{array}$ & 24 & 0 \\
\hline $\begin{array}{l}\text { Patients with } \mathrm{AUC}_{\mathrm{ss}} \text { within } \\
\text { 2nd quartile (range } \\
\text { 144,679-179,692 } \mu \mathrm{g} \cdot \mathrm{h} / \mathrm{ml} \text { ) }\end{array}$ & 24 & 0 \\
\hline $\begin{array}{l}\text { Patients with } \mathrm{AUC}_{\mathrm{ss}} \text { within } \\
\text { 3rd quartile (range } \\
181,113-216,888 \mu \mathrm{g} \cdot \mathrm{h} / \mathrm{ml} \text { ) }\end{array}$ & 23 & 2 \\
\hline $\begin{array}{l}\text { Patients with } \mathrm{AUC}_{\mathrm{ss}} \text { within } \\
\text { 4th quartile (range } \\
218,938-331,373 \mu \mathrm{g} \cdot \mathrm{h} / \mathrm{ml} \text { ) }\end{array}$ & 24 & 1 \\
\hline
\end{tabular}

$A \cup C_{S S}$ was calculated using post-hoc pharmacokinetic parameters from the final population-pharmacokinetic model. One eculizumab-treated patient for whom no posthoc pharmacokinetic parameters were obtained was excluded from the analysis.

AUC, area under the concentration-time curve within one dosing interval; ss, steady state. 
are needed in overweight patients. None of the other patient factors tested in the model significantly affected eculizumab pharmacokinetics, suggesting that dose adjustments in specific populations (for example in patients with renal/hepatic impairment) are unnecessary to maintain a favorable benefit-risk profile in patients with NMOSD.

CSF free C5 concentrations were below the lowest measurable concentration $(3.00 \mathrm{ng} / \mathrm{ml})$ after the first dose of eculizumab in all four patients in PREVENT with available data. This supports the findings of an earlier Phase 2 study in patients with AQP4-IgG-positive NMOSD in which eculizumab was shown to penetrate the CNS, with a mean (SD) CSF concentration of $34.7(18.7) \mathrm{ng} / \mathrm{ml}$ in 11 patients at 3 months of treatment (38). CSF free C5 concentrations were significantly reduced at 3 months, from a mean (SD) of $144(75.5) \mathrm{ng} / \mathrm{ml}$ in 12 patients at screening to $60.8(23.3) \mathrm{ng} / \mathrm{ml}$ in five of the 11 patients who were still receiving eculizumab at 3 months and undetectable in the other six patients (38). At present it is unknown whether free C5 suppression observed in CSF is a surrogate for complement suppression in other brain compartments where C5 activation may be involved in inducing relapses.

There was no apparent relationship between eculizumab serum concentration and anti-AQP4 antibody titer, which was to be expected as eculizumab's mechanism of action is distinct from interaction with anti-AQP4 antibodies.

There was a very low incidence in the PREVENT study population of samples testing positive for ADAs with eculizumab and no NAbs were observed. Only two patients transiently tested positive for ADA, each in one sample, both of which were negative for NAbs. This is consistent with studies of eculizumab in paroxysmal nocturnal hemoglobinuria $(\mathrm{PNH})$, atypical hemolytic uremic syndrome, and gMG $(36,40,41)$. Reducing the potential for immunogenicity was a key consideration when engineering the eculizumab molecule and was achieved by grafting the murine complementarity-determining regions into human antibody frameworks (29). The absence of NAbs indicates that sustained efficacy with long-term use of eculizumab can be expected (42).

In the PREVENT study, the incidence of AEs considered related to treatment was similar in the eculizumab and placebo groups, as was the incidence of serious AEs; most AEs were mild to moderate in severity (31). The current analysis showed no safety trends regarding AEs or AEs of special interest (infections, infusion reactions, serious cutaneous adverse reactions, cardiac disorders, and angioedema) with increasing eculizumab exposure as stratified by steady-state AUC quartiles. The membrane attack complex plays a key role in clearing Neisseria meningitidis bacteria and so blocking this pathway with eculizumab may increase the risk of meningococcal disease (23). All patients were vaccinated against $N$. meningitidis before participating in the study, and no cases of meningococcal infection were reported during the study. Neisseria meningitidis serogroups ACWY vaccination and, where available, $N$. meningitidis serogroup $B$ vaccination, are strongly recommended before initiating eculizumab therapy $(22,23)$. In an analysis of 28,518 personyears (PY) of exposure to eculizumab in the treatment of $\mathrm{PNH}$ and atypical hemolytic uremic syndrome, accumulated over almost 10 years of post-marketing pharmacovigilance

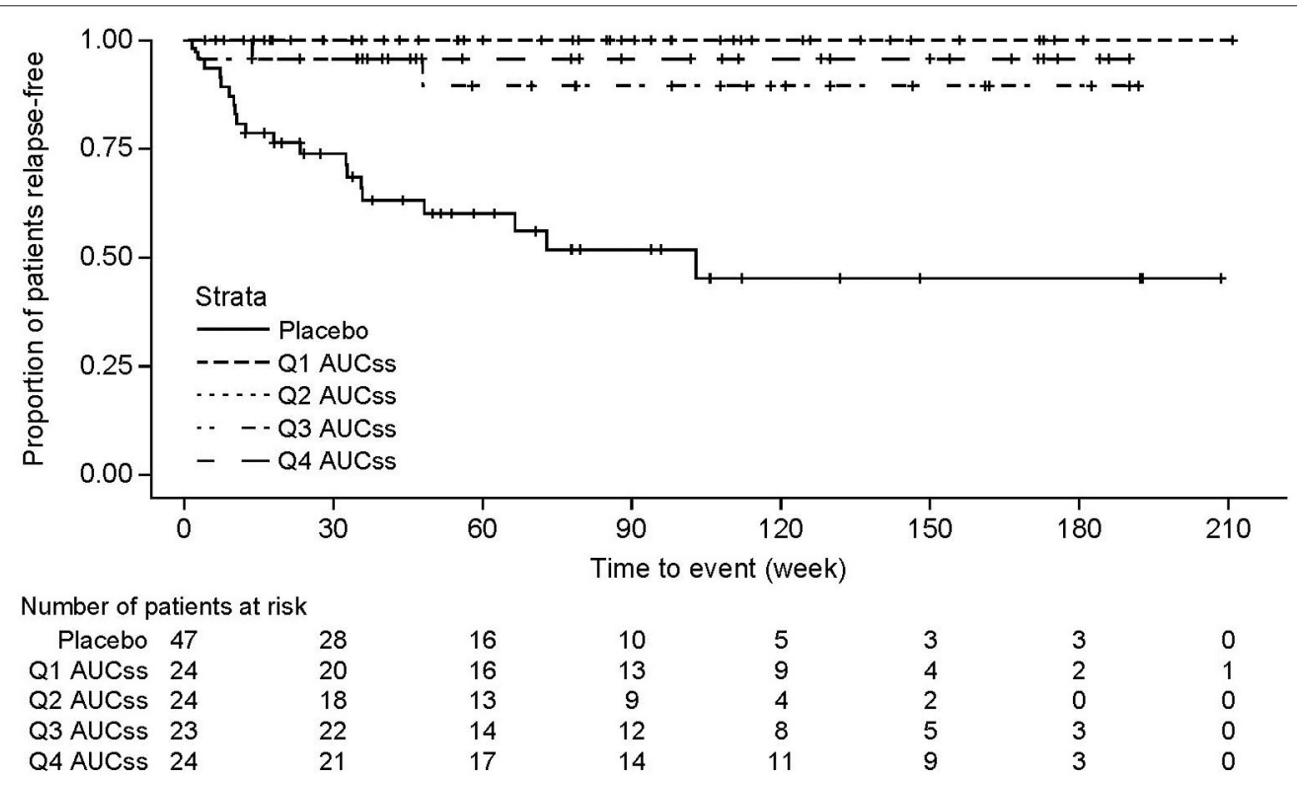

FIGURE 5 | Kaplan-Meier survival plots for time to first adjudicated on-trial relapse, according to eculizumab exposure. Relapse-free survival curves for each exposure quartile for time to first adjudicated on-trial relapse in eculizumab-treated patients. For comparison, the survival curve is also shown for patients receiving placebo. $\mathrm{AUC}_{\mathrm{sS}}$ is based on the maintenance dose of $1,200 \mathrm{mg}$ eculizumab. One eculizumab-treated patient for whom no post-hoc pharmacokinetic parameters were obtained was excluded from the analysis. AUC $\mathrm{Ss}_{\mathrm{sS}}$, area under the concentration-time curve within one dosing interval at steady state; Q1, 1st quartile; Q2, 2nd quartile; Q3, 3rd quartile; Q4, 4th quartile. 
surveillance worldwide, the overall incidence of meningococcal disease was found to be $0.25 / 100$ PY (43). As seen in the general population following introduction of the Men ACWY meningococcal vaccine in 2005, the rate of meningococcal infection in this eculizumab-treated patient population decreased over time from $0.57 / 100 \mathrm{PY}$ in 2007 to $0.16 / 100 \mathrm{PY}$ in 2016 (43).

Data on drug-drug interactions are limited, but the model used in this study incorporated concomitant medications as covariates, including systemic antibacterials, immunosuppressants, antihypertensives, and steroids. None of these medications impacted the pharmacokinetic profile of eculizumab, suggesting a lack of interaction with eculizumab. This is expected for a monoclonal antibody such as eculizumab, which is unlikely to be subject to cytochrome P450 or other enzymatic metabolism.

No differences were observed in the present analysis between Asian/non-Asian and Japanese/non-Japanese patients with regard to pharmacokinetic parameters, complete complement inhibition, or safety outcomes analyzed by eculizumab exposure.

Study limitations for this analysis include the fact that CSF data were limited owing to a very low rate of patients consenting to undergo lumbar puncture; interpretation of the CSF data should therefore be treated with caution. Data on CSF concentrations of markers and drugs are often lacking due to the invasive procedure required to collect samples; however, these (limited) CSF data from PREVENT confirm the Phase 2 study data (38). A second limitation is that only a low proportion of patients in the current analysis were Black/African American, restricting the possibility of a meaningful comparison between ethnic groups.

In summary, this rigorous quantitative assessment of the pharmacokinetic and pharmacodynamic data confirms the recommended dose regimen of eculizumab $(900 / 1,200 \mathrm{mg}$ ) has a favorable benefit-risk profile with significant clinical efficacy in patients with AQP4-IgG-positive NMOSD, without the need for dose adjustments in specific populations. Eculizumab was shown to provide rapid, complete, sustained, and well-tolerated inhibition of terminal complement activation in these patients, which translates to the significant reduction of NMOSD relapses.

\section{DATA AVAILABILITY STATEMENT}

The datasets presented in this article are not readily available; Alexion will consider requests for disclosure of clinical study participant-level data provided that participant privacy is assured through methods like data de-identification, pseudonymization, or anonymization (as required by applicable law), and if such disclosure was included in the relevant study informed consent form or similar documentation. Qualified academic investigators

\section{REFERENCES}

1. Jarius S, Ruprecht K, Wildemann B, Kuempfel T, Ringelstein M, Geis C, et al. Contrasting disease patterns in seropositive and seronegative neuromyelitis optica: a multicentre study of 175 patients. J Neuroinflammation. (2012) 9:14. doi: 10.1186/1742-2094-9-14 may request participant-level clinical data and supporting documents (statistical analysis plan and protocol) pertaining to Alexion-sponsored studies. Further details regarding data availability and instructions for requesting information are available in the Alexion Clinical Trials Disclosure and Transparency Policy at https://alexion.com/our-research/ research-and-development. Requests to access the datasets should be directed to https://alexion.com/contact-alexion/ medical-information.

\section{ETHICS STATEMENT}

The studies involving human participants were reviewed and approved by Independent Ethics Committees or Institutional Review Boards at the 70 centers that participated in the study (see Supplementary Material for full list). The patients/participants provided their written informed consent to participate in this study.

\section{AUTHOR CONTRIBUTIONS}

PS: analysis planning and strategy, data analyses and interpretation, report writing, critical review, editing, and approval of manuscript. XG: protocol design, analysis planning and strategy, data analyses and interpretation, report writing, critical review, editing, and approval of manuscript. HJK: data analysis, interpretation, report writing, critical review, editing, and approval of manuscript. FB: data analysis, interpretation, critical review, editing, and approval of manuscript. RP: bioanalyses, critical review, editing, and approval of manuscript. All authors contributed to the article and approved the submitted version.

\section{FUNDING}

Editorial assistance was provided by Duncan Porter and Jackie Mayne of Piper Medical Communications, funded by Alexion Pharmaceuticals Inc. The authors declare that this study received funding from Alexion Pharmaceuticals Inc. The funder had the following involvement with the study as sponsor: study design; collection, analysis, interpretation of data; the writing of this article; and the decision to submit it for publication.

\section{SUPPLEMENTARY MATERIAL}

The Supplementary Material for this article can be found online at: https://www.frontiersin.org/articles/10.3389/fneur. 2021.696387/full\#supplementary-material

2. Mealy MA, Boscoe A, Caro J, Levy M. Assessment of patients with neuromyelitis optica spectrum disorder using the EQ-5D. Int J MS Care. (2019) 21:129-34. doi: 10.7224/1537-2073.2017-076

3. Jiao Y, Fryer JP, Lennon VA, Jenkins SM, Quek AM, Smith CY, et al. Updated estimate of AQP4-IgG serostatus and disability outcome in neuromyelitis optica. Neurology. (2013) 81:1197-204. doi: 10.1212/wnl.0b013e3182a6cb5c 
4. Kitley J, Leite MI, Nakashima I, Waters P, McNeillis B, Brown R, et al. Prognostic factors and disease course in aquaporin-4 antibody-positive patients with neuromyelitis optica spectrum disorder from the United Kingdom and Japan. Brain. (2012) 135:1834-49. doi: 10.1093/brain/aws109

5. Wingerchuk DM, Hogancamp WF, O'Brien PC, Weinshenker BG. The clinical course of neuromyelitis optica (Devic's syndrome). Neurology. (1999) 53:1107-14. doi: 10.1212/wnl.53.5.1107

6. Wingerchuk DM, Weinshenker BG. Neuromyelitis optica: clinical predictors of a relapsing course and survival. Neurology. (2003) 60:848-53. doi: 10.1212/01.wnl.0000049912.02954.2c

7. Papadopoulos MC, Bennett JL, Verkman AS. Treatment of neuromyelitis optica: state-of-the-art and emerging therapies. Nat Rev Neurol. (2014) 10:493-506. doi: 10.1038/nrneurol.2014.141

8. Weinshenker BG, Wingerchuk DM. Neuromyelitis spectrum disorders. Mayo Clin Proc. (2017) 92:663-79. doi: 10.1016/j.mayocp.2016.12.014

9. Jarius S, Wildemann B. AQP4 antibodies in neuromyelitis optica: diagnostic and pathogenetic relevance. Nat Rev Neurol. (2010) 6:383-92. doi: 10.1038/nrneurol.2010.72

10. Kalluri SR, Illes Z, Srivastava R, Cree B, Menge T, Bennett JL, et al. Quantification and functional characterization of antibodies to native aquaporin 4 in neuromyelitis optica. Arch Neurol. (2010) 67:12018. doi: 10.1001/archneurol.2010.269

11. Lucchinetti CF, Mandler RN, McGavern D, Bruck W, Gleich G, Ransohoff RM, et al. A role for humoral mechanisms in the pathogenesis of Devic's neuromyelitis optica. Brain. (2002) 125:1450-61. doi: 10.1093/brain/awf151

12. Kuroda H, Fujihara K, Takano R, Takai Y, Takahashi T, Misu T, et al. Increase of complement fragment $\mathrm{C} 5 \mathrm{a}$ in cerebrospinal fluid during exacerbation of neuromyelitis optica. J Neuroimmunol. (2013) 254:17882. doi: 10.1016/j.jneuroim.2012.09.002

13. Levy M, Wildemann B, Jarius S, Orellano B, Sasidharan S, Weber MS, et al. Immunopathogenesis of neuromyelitis optica. Adv Immunol. (2014) 121:21342. doi: 10.1016/b978-0-12-800100-4.00006-4

14. Nytrova P, Potlukova E, Kemlink D, Woodhall M, Horakova D, Waters P, et al. Complement activation in patients with neuromyelitis optica. J Neuroimmunol. (2014) 274:185-91. doi: 10.1016/j.jneuroim.2014.07.001

15. Wang H, Wang K, Wang C, Qiu W, Lu Z, Hu X. Increased soluble C5b-9 in CSF of neuromyelitis optica. Scand J Immunol. (2014) 79:12730. doi: $10.1111 /$ sji. 12132

16. Duan T, Smith AJ, Verkman AS. Complement-dependent bystander injury to neurons in AQP4-IgG seropositive neuromyelitis optica. J Neuroinflammation. (2018) 15:294. doi: 10.1186/s12974-018-1333-z

17. Hinson SR, Romero MF, Popescu BF, Lucchinetti CF, Fryer JP, Wolburg H, et al. Molecular outcomes of neuromyelitis optica (NMO)-IgG binding to aquaporin-4 in astrocytes. Proc Natl Acad Sci U S A. (2012) 109:124550. doi: 10.1073/pnas.1109980108

18. Merle NS, Noe R, Halbwachs-Mecarelli L, Fremeaux-Bacchi V, Roumenina LT. Complement system part II: role in immunity. Front Immunol. (2015) 6:257. doi: 10.3389/fimmu.2015.00257

19. Saadoun S, Waters P, Bell BA, Vincent A, Verkman AS, Papadopoulos MC. Intra-cerebral injection of neuromyelitis optica immunoglobulin $G$ and human complement produces neuromyelitis optica lesions in mice. Brain. (2010) 133:349-61. doi: 10.1093/brain/awp309

20. Papadopoulos MC, Verkman AS. Aquaporin 4 and neuromyelitis optica. Lancet Neurol. (2012) 11:535-44. doi: 10.1016/s1474-4422(12)70133-3

21. Verkman AS. Aquaporins in clinical medicine. Annu Rev Med. (2012) 63:30316. doi: 10.1146/annurev-med-043010-193843

22. Alexion Europe SAS. Soliris (eculizumab) Summary of Product Characteristics. (2020). Available online at: https://www.medicines.org.uk/emc/product/362 (accessed January, 2021).

23. Alexion Pharmaceuticals Inc. SOLIRIS ${ }^{\circledR}$ (eculizumab) Injection Prescribing Information. (2020). Available online at: https://alexion.com/Documents/ Soliris_USPI.pdf (accessed January, 2021).

24. Frampton JE. Eculizumab: a review in neuromyelitis optica spectrum disorder. Drugs. (2020) 80:719-27. doi: 10.1007/s40265-020-01297-w

25. Health Canada. SOLIRIS (eculizumab) Product Information. (2019). Available online at: https://pdf.hres.ca/dpd_pm/00053762.PDF (accessed January, 2021).
26. Pharmaceuticals and Medical Devices Agency. Eculizumab (Genetical Recombination) SOLIRIS ${ }^{\circledR}$ for Intravenous Infusion $300 \mathrm{mg}$ (Japanese Package Insert). 2nd ed. (2020). Available online at: https://www.pmda.go.jp/ PmdaSearch/iyakuDetail/ResultDataSetPDF/870056_6399424A1023_1_16 (accessed January, 2021).

27. Therapeutic Goods Administration. SOLIRIS Australian Prescribing Information. (2020). Available online at: https://www.ebs.tga.gov.au/ebs/ picmi/picmirepository.nsf/pdf?OpenAgent\&id=CP-2010-PI-02947-3\&d= 202101061016933 (accessed January, 2021).

28. Thomas TC, Rollins SA, Rother RP, Giannoni MA, Hartman SL, Elliott EA, et al. Inhibition of complement activity by humanized anti-C5 antibody and single-chain Fv. Mol Immunol. (1996) 33:1389-401. doi: 10.1016/s0161-5890(96)00078-8

29. Rother RP, Rollins SA, Mojcik CF, Brodsky RA, Bell L. Discovery and development of the complement inhibitor eculizumab for the treatment of paroxysmal nocturnal hemoglobinuria. Nat Biotechnol. (2007) 25:125664. doi: $10.1038 /$ nbt1344

30. Wijnsma KL, ter Heine R, Moes DJAR, Langemeijer S, Schols SEM, Volokhina EB, et al. Pharmacology, pharmacokinetics and pharmacodynamics of eculizumab, and possibilities for an individualized approach to eculizumab. Clin Pharmacokinet. (2019) 58:859-74. doi: 10.1007/s40262-019-00742-8

31. Pittock SJ, Berthele A, Fujihara K, Kim HJ, Levy M, Palace J, et al. Eculizumab in aquaporin-4-positive neuromyelitis optica spectrum disorder. N Engl J Med. (2019) 381:614-25. doi: 10.1056/nejmoa19 00866

32. World Medical Association. WMA Declaration of Helsinki - Ethical Principles for Medical Research Involving Human Subjects. Ferney-Voltaire: World Medical Association (2013). Available online at: https://www.wma.net/ policies-post/wma-declaration-of-helsinki-ethical-principles-for-medicalresearch-involving-human-subjects/ (accessed May, 2021).

33. International Conference on Harmonisation of technical requirements for registration of pharmaceuticals for human use. ICH harmonized tripartite guideline: Guideline for Good Clinical Practice. J Postgrad Med. (2001) 47:45-50.

34. United States Food and Drug Administration. Guidance for Industry: Bioanalytical Method Validation. Rockville, MD: US Department of Health and Human Services (2001).

35. European Medicines Agency Committee for Medicinal Products for Human Use. Guideline on Bioanalytical Method Validation. London: European Medicines Agency (2011). Available online at: https://www.ema.europa. eu/en/documents/scientific-guideline/guideline-bioanalytical-methodvalidation_en.pdf (accessed January, 2021).

36. Monteleone JPR, Gao X, Kleijn HJ, Bellanti F, Pelto R. Eculizumab pharmacokinetics and pharmacodynamics in patients with generalized myasthenia gravis. Front Neurol. (2021). doi: 10.3389/fneur.2021.69 6385

37. Howard JF, Jr., Utsugisawa K, Benatar M, Murai H, Barohn RJ, Illa I, et al. Safety and efficacy of eculizumab in anti-acetylcholine receptor antibody-positive refractory generalised myasthenia gravis (REGAIN): a phase 3, randomised, double-blind, placebo-controlled, multicentre study. Lancet Neurol. (2017) 16:976-86. doi: 10.1016/s1474-4422(17)30 369-1

38. Pittock SJ, Lennon VA, McKeon A, Mandrekar J, Weinshenker BG, Lucchinetti CF, et al. Eculizumab in AQP4-IgG-positive relapsing neuromyelitis optica spectrum disorders: an open-label pilot study. Lancet Neurol. (2013) 12:554-62. doi: 10.1016/s1474-4422(13)70 076-0

39. Dhillon S. Eculizumab: a review in generalized myasthenia gravis. Drugs. (2018) 78:367-76. doi: 10.1007/s40265-018-0875-9

40. Greenbaum LA, Fila M, Ardissino G, Al-Akash SI, Evans J, Henning $P$, et al. Eculizumab is a safe and effective treatment in pediatric patients with atypical hemolytic uremic syndrome. Kidney Int. (2016) 89:701-11. doi: 10.1016/j.kint.2015. 11.026

41. Hillmen P, Muus P, Szer J, Hill A, Höchsmann B, Kulasekararaj A, et al. Assessment of human antihuman antibodies to eculizumab after long-term treatment in patients with paroxysmal nocturnal 
hemoglobinuria. Am J Hematol. (2016) 91:E16-7. doi: 10.1002/ajh. 24280

42. Kuriakose A, Chirmule N, Nair P. Immunogenicity of biotherapeutics: causes and association with posttranslational modifications. $J$ Immunol Res. (2016) 2016:1298473. doi: 10.1155/2016/129 8473

43. Socié G, Caby-Tosi MP, Marantz JL, Cole A, Bedrosian CL, Gasteyger C, et al. Eculizumab in paroxysmal nocturnal haemoglobinuria and atypical haemolytic uraemic syndrome: 10-year pharmacovigilance analysis. $\mathrm{Br} \mathrm{J}$ Haematol. (2019) 185:297-310. doi: 10.1111/bjh.15790

Conflict of Interest: PS and XG were employees of Alexion Pharmaceuticals, Inc. at the time the work described in this paper was undertaken; RP is an employee of Alexion Pharmaceuticals, Inc.; HJK and FB are employees of Certara Strategic Consulting, which received funding from Alexion Pharmaceuticals.

The authors declare that this study received funding from Alexion Pharmaceuticals Inc. The funder had the following involvement with the study as sponsor: study design; collection, analysis, interpretation of data, the writing of this article, and the decision to submit it for publication. Editorial assistance was provided by Piper Medical Communications, funded by Alexion Pharmaceuticals Inc.

Publisher's Note: All claims expressed in this article are solely those of the authors and do not necessarily represent those of their affiliated organizations, or those of the publisher, the editors and the reviewers. Any product that may be evaluated in this article, or claim that may be made by its manufacturer, is not guaranteed or endorsed by the publisher.

Copyright $\odot 2021$ Singh, Gao, Kleijn, Bellanti and Pelto. This is an open-access article distributed under the terms of the Creative Commons Attribution License (CC $B Y)$. The use, distribution or reproduction in other forums is permitted, provided the original author(s) and the copyright owner(s) are credited and that the original publication in this journal is cited, in accordance with accepted academic practice. No use, distribution or reproduction is permitted which does not comply with these terms. 